\title{
Annual Variation of Local Photon Emissions' Spectral Power within the mHz Range Overlaps with Seismic-Atmospheric Acoustic Oscillations
}

\author{
Michael A. Persinger \\ Laurentian University, Sudbury, Canada \\ Email: mpersinger@laurentian.ca
}

Received November 14, 2011; revised December 23, 2011; accepted January 25, 2012

\begin{abstract}
Spheroidal modes of seismic and acoustic oscillations in the atmosphere occur within the 2 to $7 \mathrm{mHz}$ range with peakto-peak variations in the order of $10^{-12}$ to $10^{-11} \mathrm{~m} \cdot \mathrm{s}^{-2}$. Previous research indicated the amplitudes for $230 \mathrm{~s}$ and $270 \mathrm{~s}$ periods peak during the summer months. In the present study the amplitudes of a reliably apparent $3 \mathrm{mHz}$ increment from spectral analyses of minute-to-minute measurements of background photon emissions by a photomultiplier tube housed in a dark room were sampled for a one year period. The peak increase in the power of this increment was maximal during the summer months and overlapped conspicuously with the annual variation in fundamental spheroidal modes of seismic free oscillations. Quantitative estimates indicate that relative shifts in the order of $10^{-11} \mathrm{~W} / \mathrm{m}^{2}$ for photon emissions may reflect the annual variation in coupled oscillations between the earth and atmosphere.
\end{abstract}

Keywords: Photon Emissions; Earth Oscillations; Spheroidal Modes; Periodicity; Annual Variations

\section{Introduction}

Fundamental spheroidal modes or background free oscillations within the earth have been firmly established [1]. They occupy a relatively wide band, primarily within the 2 - $7 \mathrm{mHz}$ range [2], whose general peak-to-peak amplitudes are $\sim 0.5 \mathrm{nGal}$ (nanogalileos) where $1 \mathrm{nGal}=$ $10^{-11} \mathrm{~m} \cdot \mathrm{s}^{-2}$. This "earth hum" originates in the Pacific and southern oceans depending upon season [3]. The sources of these oscillations are not likely to be averaged forces from cumulative amplitudes of many small earthquakes. According to Nishida et al. [3] the excitation source is not within the solid earth but emerges at the boundary between the earth's surface and the atmosphere.

Within this interface seismic free oscillations resonate with acoustic free oscillations of the atmosphere. These authors reported annual oscillations in the amplitude of Raleigh waves, particularly around $230 \mathrm{~s}(4.3 \mathrm{mHz})$ and $270 \mathrm{~s}(3.7 \mathrm{mHz})$, over a nine year period. These peaks corresponded to fundamental spheroidal mode ${ }_{0} \mathrm{~S}_{29}$ and ${ }_{0} S_{37}$, respectively. The annual variation in amplitude ranged from 0.4 to $0.6 \mathrm{nGal}$ with a peak during the summer months. The equivalent annual variation in infrared flux, which overlapped significantly with the annual variations in model amplitudes, ranged from about 232 to $242 \mathrm{~W} / \mathrm{m}^{2}$. The coincidence was interpreted as evidence that the dynamic pressure from some source within the atmosphere excites the Earth's free oscillations.
For the last three years minute-to-minute measures of background photon emission have been recorded by the station's (Sudbury, Ontario) photomultiplier tube (PMT). We [4] have found conspicuous increases in photon emission several days before the major $(M>8.0)$ global earthquakes in Japan and Chile during the last two years and smaller but statistically significant increases in background photon emission during the days before $7.0<$ $\mathrm{M}<8.0$ seismic events. To discern if more subtle changes in photon emissions occurred over the year, spectral analyses of the power of photon emissions were sampled. A $3 \mathrm{mHz}$ peak in power was consistently noted in daily records whose amplitudes changed systematically over months. Here I report a remarkably similar annual variation the amplitude of power within a specific frequency similar to the frequency reported by Nishida $e t$ al. [3] for free earth oscillations.

\section{Methods and Materials}

A model 15 Photometer from SRI Instruments (Pacific Photometric Instruments) with a PMT housing (BCA IP21) for a RCA electron tube (no filters) has been housed in the same locus for over three years. It is located in a dark room in the basement of the Classroom Building at Laurentian University. The PMT is connected to a digital voltmeter; continuously fluctuating values are sampled and stored once per min, 24 hr/ day by a IBM ThinkPad 
laptop (Windows 95) computer (i.e., total of 1440 samples/ day). Calibration by several methods indicated that 1 unit of change along the 1 to 100 unit PMT scale is approximately $5 \times 10^{-11} \mathrm{~W} / \mathrm{m}^{2}$ when referenced to the midrange, i.e., 50 units [5]. The data for the quietist day, judged by visual inspection of printed daily records, and for the day when global seismic activity was minimal for several days before and afterwards was extracted for each month between July 2009 and June 2010. Spectral analyses were completed by SPSS PC-16 software and Plotter for con- firmation. The relative power for the $3 \mathrm{mHz}$ peak, that ranged from 0.2 to 0.6 , was obtained for each month. The power was superimposed over the annual variation in free oscillations and infrared flux in Figure 2B of Nishada et al's data [3] that were replotted for this paper. Because the PMT measurements began in July 2009 the first part of the year was for the following year 2010 .

\section{Results}

The relative spectral power for the $3 \mathrm{mHz}$ peak over the 12 months (January $=1$ ) is indicated by the large black circles in Figure 1. The solid square $\left({ }_{0} \mathrm{~S}_{20}\right)$ and thin solid line $\left({ }_{0} \mathrm{~S}_{45}\right)$ reflect the 9 year data averages for free earth oscillations from Nishada et al. [3]; the vertical bars were their fitting errors. The circles and dashed line indicate the infrared flux at the top of the atmosphere. It is clear that the annual variation of the spectral power for $3 \mathrm{mHz}$ oscillation in background photon emissions peaked during the summer months (for that year) and overlapped with the amplitude variations of earth oscillations.

\section{Discussion and Implications}

There is classic theoretical and empirical evidence that photon emissions can originate from the types of silicates contained within crustal structures. Presumably the source

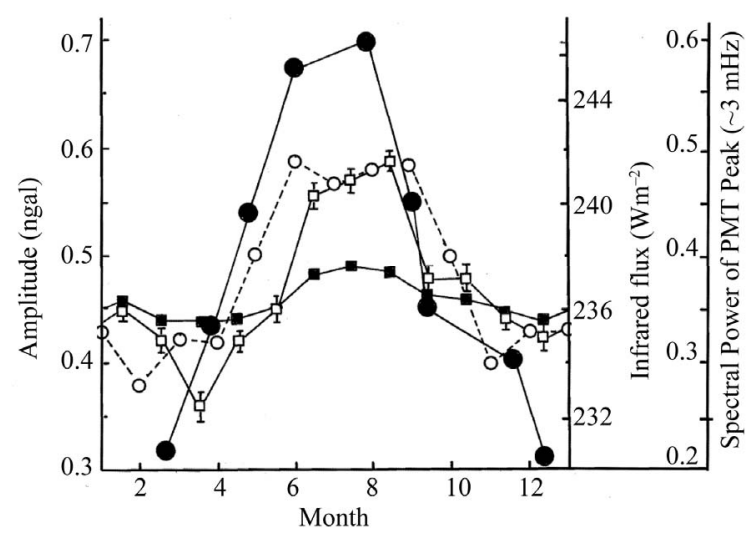

Figure 1. Monthly amplitudes in ngals $\left(10^{-11} \mathrm{~m} \cdot \mathrm{s}^{-2}\right)$ for earth oscillations in two modes (open circles and squares) and infrared flux densities (solid squares) from Nishada et al. in comparison with the spectral powers of photon emission oscillations (large solid circles) around $3 \mathrm{mHz}$. of such emissions would be subtle mechanical pressures or electromagnetic stimuli. According to Nishada et al. [3] dynamic pressure of atmospheric origin is a likely major source to excite Earth's free oscillations. One particular conspicuous spectral frequency, around $3 \mathrm{mHz}$, from measurements for one year of background photon emissions in a darkened basement room in the Sudbury Basin displayed an annual variation that was remarkably similar to the amplitudes of both free earth oscillations and infrared flux density.

For Nishada et al's [3] data the amplitude of variation for the major ${ }_{0} \mathrm{~S}_{29}$ mode was $40 \%$ with the remaining modes about $10 \%$. In comparison the infrared flux density variation was about 5\%. The amplitude of variation for the photon emission amplitudes at $3 \mathrm{mHz}$ would be more approximate because of the relative measures. However considering the peak higher frequencies with values around 2.0 to 2.5 , the range of 0.4 relative power units would be equivalent to a variation between $16 \%$ and $20 \%$. With a mean background PMT measurement of $5 \times 10^{-11} \mathrm{~W} / \mathrm{m}^{2}$ and $+/-2$ standard deviations (range) of $\sim 5$ units during a quiet period over a $24 \mathrm{hr}$ interval, this would be equivalent to between 1 and $2 \times 10^{-11} \mathrm{~W} / \mathrm{m}^{2}$. For comparison the equivalent magnetic energy derived from $B^{2}=J 2 \mu / m^{3}$ would be about $5 \mathrm{nT}$. This magnitude is similar to geomagnetic pulsations arising in the earth-solar wind environment at surface midlatitude locations and is also within the range of the mean value for the solar wind [6].

Although the effects of subtle geophysical forces and energies upon the human observer are often not considered in traditional geophysics, the $0.5 \mathrm{nGal}\left(0.5 \times 10^{-11} \mathrm{~m} / \mathrm{s}^{2}\right)$ free oscillations may be more important than assumed. For a $70 \mathrm{~kg}$ mass (the average human being), the resulting force would be $3.5 \times 10^{-10} \mathrm{~N}$ which is within the range of forces for cell-to-cell adhesion [7]. When applied to the volume of a person with a cross section of $0.25 \mathrm{~m}^{2}$ the resulting pressure would be $\sim 1.4 \times 10^{-9} \mathrm{~Pa}$ which is remarkably similar to the averaged universal pressure [8]. When multiplied by the person's volume (assuming $7 \times 10^{-2}$ $\mathrm{m}^{3}$ ) the energy would be $9.8 \times 10^{-11} \mathrm{~J}$. If the variation was $1 \mathrm{~Hz}$, the power density would be $\sim 3.9 \times 10^{-10} \mathrm{~W} / \mathrm{m}^{2}$. Interestingly, this is the same order of magnitude as "spontaneous" photon emission from the right hemisphere of cerebrums during visualization of light by some darkadapted human volunteers sitting in the dark [9] and cell cultures when removed from optimal thermal environments [5].

If the temporal distribution of the energy change involved the $270 \mathrm{~s}$ or $230 \mathrm{~s}$ periods from the earth oscillations the energy would be in the range of 1.4 to $1.7 \times$ $10^{-12} \mathrm{~W} / \mathrm{m}^{2}$ which is within the range generated by tissue slices from the hippocampus [10], the area of the brain involved with memory consolidation. Interesting, the photon emissions measured from the body surface are $\sim 10^{7}$ 
photons $/ \mathrm{s} \cdot \mathrm{m}^{2}$ [11]. Assuming the emitted photons are within the visible wavelengths, each with the unit energy of $10^{-19} \mathrm{~J}$, the resulting power density is within the order of $10^{-12} \mathrm{~W} / \mathrm{m}^{2}$. Traditionally calculated radial currents values from the spherical harmonic coefficients for the non-potential geomagnetic field within which seismic and atmospheric oscillations are immersed are in the order of $10^{-9} \mathrm{~A} / \mathrm{m}^{2}$ [12] and when applied across potential differences of $10^{-3} \mathrm{~V}$, the interface between the domains of cerebral steady potential shifts and electroencephalographic activity, would be $\sim 10^{-12} \mathrm{~W} / \mathrm{m}^{2}$.

\section{Acknowledgements}

Thanks to Professors Stanley Koren and Blake T. Dotta and Mr. Brendan Lehman for technical assistance.

\section{REFERENCES}

[1] T. Tanimoto, J. Ulm, K. Nishida and N. Kobayashi, "Earth’s Continuous Oscillations Observed on Seismically Quiet Days,” Geophysical Letters, Vol. 25, No. 10, 1998, pp. 15531563. doi:10.1029/98GL01223

[2] J. Rhie and B. Romanowicz, "Excitation of Earth's Continuous Free Oscillations by Atmospheric-Ocean-Seafloor Coupling,” Nature, Vol. 431, 2004, pp. 552-555. doi:10.1038/nature02942

[3] K. Nishida, N. Kobayashi and Y. Fukao, "Resonant Oscillations between the Solid Earth and the Atmosphere," Science, Vol. 287, No. 5461, 2000, pp. 2244-2246. doi:10.1126/science.287.5461.2244

[4] M. A. Persinger, B. T. Dotta and G. F. Lafreniere, "Marked Increases in Background Photon Emissions in Sudbury On- tario More than One Week before the Magnitude > 8.0 Earthquakes in Japan and Chile,” in submission.

[5] B. T. Dotta, C. A. Buckner, D. Cameron, R. M. Lafrenie and M. A. Persinger, "Biophoton Emissions from Cell Cultures: Biochemical Evidence for the Plasma Membrane as the Primary Source," General Physiology and Biophysics, Vol. 30, No. 4, 2011, pp. 301-309.

[6] W. H. Campbell, "Introduction to Geomagnetic Fields," Cambridge University Press, Cambridge, 1997.

[7] A. D. Bershadky, N. Q. Balaban and B. Geiger, "Adhesion-Dependent Cell Mechanosensitivity,” Annual Reviews of Cell Developmental Biology, Vol. 19, 2003, pp. 677-695.

[8] M. A. Persinger, "A Simple Estimate for the Mass of the Universe: Dimensionaless Parameter A and the Construct of 'Pressure'," Journal of Physics, Astrophysics and Physical Cosmology, Vol. 3, No. 1, 2010, pp. 1-3.

[9] B. T. Dotta, C. A. Buckner, R. M. Lafrenie and M. A. Persinger, "Photon Emissions From Human Brain and Cell Culture Exposed to Distally Rotating Magnetic Fields Shared by Separate Light-Stimulated Brains and Cells,” Brain Research, Vol. 1388, 2011, pp. 77-88. doi:10.1016/j.brainres.2011.03.001

[10] Y. Isojima, T. Isoshima, K. Nagai, K. Kikuchi and H. Nakagawa, "Ultraweak Biochemiluninescence Detected from Rat Hippocampal Slices,” NeuroReports, Vol. 6, No. 4, 1995, pp. 658-660. doi:10.1097/00001756-199503000-00018

[11] F. A. Popp, "Electromagnetic Information,” Urban and Schwartzberg, New York, 1979, pp. 543-544.

[12] D. E. Winch, D. J. Ivers, J. P. R. Turner and R. J. Stening, "Geomagnetism and Schmidt Quasi-Normalization," Geophysical Journal International, Vol. 160, No. 2, 2005, pp. 487-504. doi:10.1111/j.1365-246X.2004.02472.x 